\title{
Chronic otitis media progressing to temporal bone osteomyelitis and sequestration with mastoid cavity myiasis in paediatric age group: an unusual case report
}

\author{
Prashanth Kudure Basavaraj, Manjunatha H. Anandappa*, Nishtha Sharma, \\ Shreyas Karkala, Veena Prabhakaran
}

Department of ENT, JJM Medical College, Davangere, Karnataka, India

Received: 03 February 2020

Revised: 12 March 2020

Accepted: 17 March 2020

\author{
*Correspondence: \\ Dr. Manjunatha H. Anandappa, \\ E-mail: hamanjuha@gmail.com
}

Copyright: (C) the author(s), publisher and licensee Medip Academy. This is an open-access article distributed under the terms of the Creative Commons Attribution Non-Commercial License, which permits unrestricted non-commercial use, distribution, and reproduction in any medium, provided the original work is properly cited.

\begin{abstract}
Myiasis is the infestation by fly larvae. There are few sporadic publications regarding human ear myiasis. Osteomyelitis of temporal bone is also an uncommon entity. This study aims to describe the clinical presentation and management of a unique presentation of mastoid cavity myiasis and sequestration in a 4 year old child, who presented to Chigateri district hospital, Davangere with blood tinged discharge along with visible maggots from left external auditory canal and post auricular sinus since 3 days, with history of chronic otitis media. After manual cleaning of maggots, high resonance computed tomography of temporal bone was done, which showed findings suggestive of foreign body with bony density. Modified radical mastoidectomy was done, where osteomyelitis of temporal bone with sequestrum was encountered on table and removed. Diagnosis of temporal bone osteomyelitis requires a high degree of suspicion, especially in a setting of myiasis, as these 2 rare pathologies have not been reported to co-occur in literature. Early intervention is essential to avoid fatal complications of this infrequent presentation.
\end{abstract}

Keywords: Chronic otitis media, Myiasis, Sequestrum, Osteomyelitis, Post-aural fistula

\section{INTRODUCTION}

Myiasis is the infestation of humans by fly larvae, which feed of the host's healthy or diseased organs and tissues. There are few sporadic publications regarding human ear myiasis.

Osteomyelitis is a chronic inflammatory process affecting bones and marrow cavities. Usually seen in the long bones as a result of trauma and/or presence of co-morbid conditions, osteomyelitis of temporal bone happens to be an uncommon entity.

The purpose of this article is to describe the clinical picture and management of a unique presentation of mastoid cavity myiasis and sequestration in a four years old child. What makes this case unique is not only the unusual age of presentation of an osteomyelitic process, but also the coexistence of these two rare conditions, with this being the first case to be reported in literature.

\section{CASE REPORT}

A four years old male child belonging to low socioeconomic stratum presented to the emergency department of Chigateri General Hospital, Davangere, Karnataka, India with excruciating left ear pain of three days duration, along with blood tinged discharge and visible maggots in the left external auditory canal. Patient's father also complained of a left post auricular sinus in the past three days, with similar discharge and maggots coming out of it. There was history of left chronic otitis 
media in the past three years, with recurrent episodes of mucoid discharge from the left ear, subsiding temporarily on medication. One month prior to presenting to our hospital, patient had started to develop a gradually progressive painful swelling in the left mastoid region. There was no history of fever or trauma to the ear, nor was there any complaint of facial deviation.

The patient was taken to a nearby hospital on the second day of this presentation, where turpentine oil was instilled and some of the maggots were removed. The patient was started on intravenous antibiotics, and was then referred to our hospital for further management of the case.

On examination, the child looked alert but irritable, and was found to have stable vital signs. He was afebrile at the time of presentation. Examination of the left ear revealed foul-smelling blood-stained discharge. On performing aural toileting, an irregular hard bulge was felt on the posterior canal wall with grating sensation. Discharge and maggots present medial to this bulge could not be cleaned and hence tympanic membrane could not be visualized. The left post auricular region had an open mastoid sinus with diameter of $0.5 \mathrm{~cm}$, in which foul smelling sero-sanguinous discharge was seen along with multiple visible maggots. The surrounding skin appeared stretched, shiny and erythematous, and tenderness was elicited on palpation. The patient was admitted, and after daily instillation of turpentine oil at night to eradicate the maggots and regular toileting, the mastoid sinus was seen to be deep, lined with granulation tissue with a bony overgrowth in the inferior part. The rest of the ENT examination was found to be within normal limits.
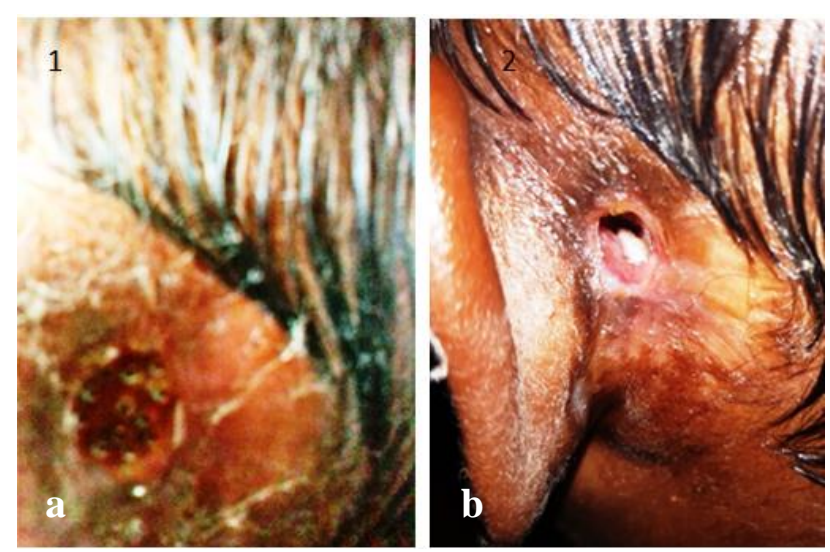

Figure 1: Clinical picture of myiasis and osteomyelitis of temporal bone causing post auricular sinus, (a) visible maggots in left post auricular sinus and (b) bony overgrowth in inferior part with granulation tissue lining the walls of sinus, seen after eradication of maggots.

High resolution computed tomography (HRCT) of temporal lobe was performed for the child, which revealed an old fracture of the left mastoid with a fracture fragment impacted in left external auditory canal. There was surrounding soft tissue edema, with collection in the overlying scalp and periauricular soft tissues. Ossicular chain was not well visualized. Sclerosis of mastoid air cells was found, but mastoid antrum was not separately seen. CT scan of brain showed no abnormal attenuation in neuroparenchyma, hence ruling out any intracranial extension. Hyperdense ossified focal lesion surrounded by soft tissue was seen impacted in left external auditory canal, with edema and fluid around the left external auditory canal and abscess formation in the left periauricular region. Complete blood counts, liver and renal function test and coagulation profile was found to be normal.
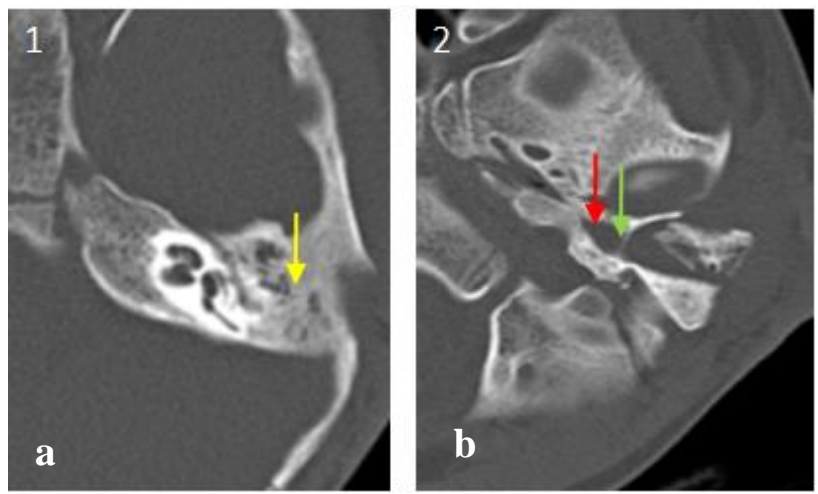

Figure 2: HRCT temporal bone images of patient having chronic otitis media with maggot infestation, (a) sclerosis of left mastoid air cells (shown by yellow arrow) and (b) intact tympanic membrane (shown by green arrow) with fluid collection behind it (shown by red arrow).

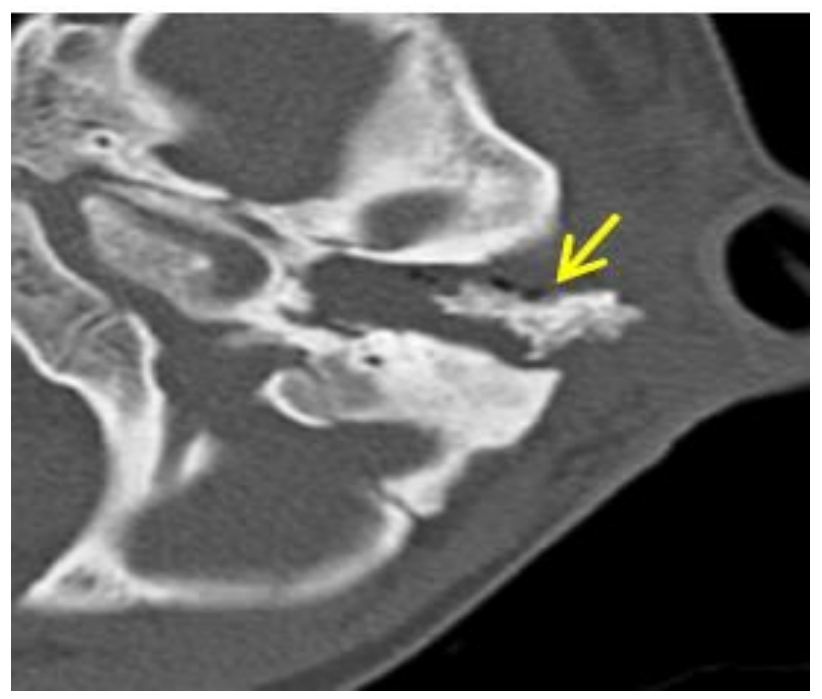

Figure 3: HRCT temporal bone showing bony opacity, suspected to be sequestrum, in the left temporal bone (shown by yellow arrow).

Patient was started on intravenous antibiotics, namely meropenem, piperacillin-tazobactam and metrogyl in appropriate dosage by weight after taking a paediatric consultation, and was also given intravenous steroids to subside the inflammation. After three days of regular 
aural toileting and manual removal of maggots after instilling turpentine oil, all larvae were eradicated.

Unconvinced with the findings of impacted fracture fragment and foreign body etiology on imaging, a modified radical mastoidectomy under general anesthesia was planned for the left ear. On evaluating under microscope in the operation theatre, post aural sinus opening was present, which was seen to be communicating with the left external auditory canal. Post aural incision was given with an elliptical extension around the sinus opening. Bony sequestrum was found in the mastoid cavity, which was removed to reveal cavity lined by granulation tissue. This tissue was removed with a diamond drill bit until healthy bone was obtained. Thick intact tympanic membrane was then visualized, which was elevated to inspect the middle ear cavity. No middle ear landmarks were present in the patient. The tympanic membrane was repositioned, temporalis fascia graft was harvested and placed over the mastoid cavity and absorbable gel foam placed. The skin was sutured along the elliptical incision to obliterate sinus opening and mastoid dressing was done.
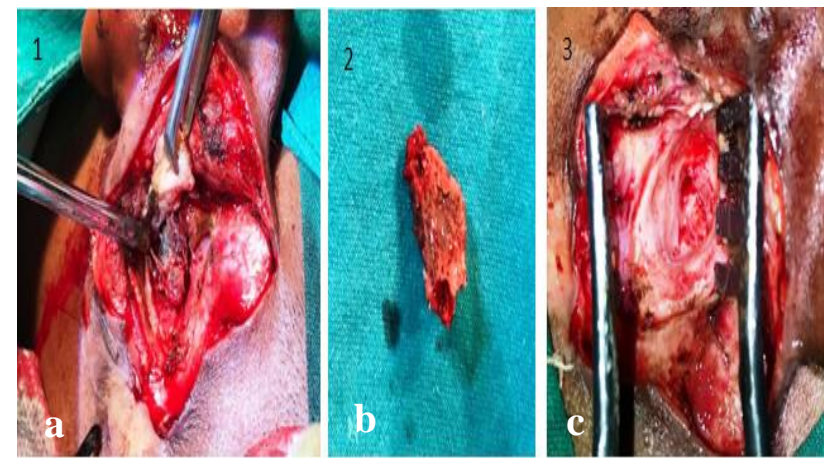

Figure 4: Intraoperative images of left modified radical mastoidectomy, (a) bony sequestrum found in mastoid cavity, (b) bony sequestrum after removal from mastoid cavity, and (c) mastoid cavity after clearance of granulation tissue.

Post operatively, intravenous antibiotics and steroids were continued along with analgesics, and patient was discharged on the third post-operative day after switching to oral antibiotics. Patient was reviewed on the tenth postoperative day to obtain a healthy post aural wound, and sutures were removed. External auditory canal contained gel foam, for which topical antibiotic drops were instilled regularly. Patient had regular monthly follow-ups, where he was found to be asymptomatic, having a healthy scar and mastoid cavity. Patient has been advised a second stage surgery for ossicular reconstruction in the future.

\section{DISCUSSION}

Osteomyelitis is the inflammation of bones due to an impairment of vascularity and the resultant infection of the marrow cavity. Due to compromised blood flow to the periosteum, it gradually loses its nutrient supply and becomes necrotic, forming a dead bone called 'sequestrum', which is the pathogenomic feature of this condition. New bone formation may occur around the area of infection in the later stage of the disease, which is known as 'involucrum'. While it is known to occur in the long bones such as the tibia, femur etc following trauma or in the setting of immunocompromised health state or uncontrolled diabetes mellitus, it is not commonly seen to affect the temporal bone.

Indeed, temporal bone osteomyelitis is an infrequently encountered entity. Toulmouche was the first to report a case of temporal bone osteomyelitis in $1838 .{ }^{1}$ Known to occur most commonly in those suffering from diabetes mellitus or malnourishment, temporal bone osteomyelitis finds its predisposition in patients affected by certain diseases of the ear such as trauma to the bone, chronic suppurative otitis media, mastoiditis, otitis externa, tuberculosis or even certain malignancies impeding the vasculature of the temporal bone.

Patients, mostly old aged with a background of diabetes, present with deep seated pain and edema over the mastoid region, with malodorous suppuration from the ear. In some extensive cases, cranial nerves may get involved. Imaging studies may reveal granulation tissue and micro abscesses in the temporal bone. Most common organism isolated from the ear discharge of patients of temporal bone osteomyelitis is Pseudomonas aeruginosa. All of the above-mentioned features were suggested as the criteria for diagnosis by Anderhuber et al after their extensive study of this disease. ${ }^{2}$

While HRCT of the temporal bone is the most commonly used imaging modality for diagnosing osteomyelitis of temporal bone due to its ability to delineate the extent of the disease process, MRI has an advantage in picking intracranial spread of the disease. Gallium scan can be done to monitor the presence of infection. ${ }^{2}$

Treatment of temporal bone osteomyelitis lies not only in the targeted medical management of the organism involved with appropriate antibiotics and the control of blood sugar levels, but also in the prompt debridement and surgical clearance of the disease completely from the bone.

The term myiasis comes from the Greek word 'myia' which means 'fly'. ${ }^{3}$ Hence, myiasis is the invasion of Dipteran two winged flies or their larvae into humans or other vertebrate animals leading to infection of the infested body tissue. ${ }^{3}$ According to case reports across the world, the families Calliphoridae, Oestridae and Sarcophagidae are implicated to cause myiasis in humans. ${ }^{4}$

It is easy to understand that people of low socioeconomic stratum, living in the rural areas with poor hygiene and nutrition would be more prone to developing 
myiasis. Inability to ward off these flies regularly is an additional risk factor, thus affecting children, mentally challenged adults or delibitated individuals. Any ongoing inflammatory process can also serve as a bed for these larvae to grow and multiply.

Aural myiasis is a rare condition, with sparce reports on the same published in text. Even more uncommon is the occurrence of myiasis in the mastoid region, and those present are reported to develop in patients having undergone mastoidectomy in the recent past. We have failed to come across more than two case reports claiming myiasis affecting temporal bone untouched by trauma or surgery, one of them being in Belgaum in 1925, and the more recent one reported in Saudi Arabia in $2003 .^{5,4}$

Patients suffering from aural myiasis complain of severe pain in the ear along with foul smelling blood stained purulent discharge. The patient may experience a foreign body sensation in the ear. Some may also have giddiness and hearing impairment. On examination, maggots or their eggs may be visualised in a background of ear discharge and granulation. ${ }^{3}$ What makes this condition dangerous is the possibility of the larvae migrating to the brain leading to fatal intracranial complications, with mortality reaching up to eight percent. ${ }^{3}$

Hence, treatment should be prompt and effective. While instillation of topical agents like turpentine oil are known to kill the maggots, the eggs remain unaffected and continue to hatch, hence daily inspection and manual removal of maggots is essential to ensure complete clearance. Antibiotic cover should be given to control the underlying infection. Surgical intervention should be considered to remove the granulation tissue if medical treatment does not seem effective.

Our study is the first to report the co-occurrence of temporal bone osteomyelitis with mastoid cavity myiasis, both being rare independent of each other. Not only is it uncommon for a child to develop osteomyelitis considering the absence of trauma or diabetes, simultaneous presence of visible myiasis made the diagnosis a difficult one, especially with imaging results misleading us to suspect an ossified foreign body or a fracture fragment. We suspect the patient to have developed osteomyelitis as a result of chronic unresolved otitis media and mastoiditis, with the superimposed myiasis causing the mastoid cavity sinus to develop.
Whether the erosion of posterior wall of external auditory canal and its communication to the mastoid cavity was the result of maggot infestation, or the cause for their invasion to the temporal bone, remains a dilemma.

\section{CONCLUSION}

Diagnosis of temporal bone osteomyelitis requires a high degree of suspicion, especially in a setting of myiasis, as these two rare pathologies have not been reported to coexist in literature. Early intervention is essential to avoid fatal complications of this infrequent presentation.

\section{ACKNOWLEDGEMENTS}

We are thankful to the department of ENT, JJM medical college, Davangere, and Chigateri district hospital, Davangere for their support.

Funding: No funding sources

Conflict of interest: None declared

Ethical approval: Not required

\section{REFERENCES}

1. Gyawali BR, Rayamajhi P. Osteomyelitis of temporal bone: A case report of a rare disease. Indian J Otol. 2018;24:114-6.

2. Prasad SC, Prasad KC, Kumar A, Thada ND, Rao P, Chalasani S. Osteomyelitis of the temporal bone: terminology, diagnosis, and management. J Neurol Surg B Skull Base. 2014;75(5):324-31.

3. Gulia JS, Yadav SPS, Khaowas A. Mastoid cavity myiasis in a child: A case report. Int $\mathrm{J}$ Tropical Med. 2010;6(2):4.

4. Abidi AA, Bello C, Ahmari AM, Fawehinmi Y. Mastoid cells myiasis in a Saudi man: A case report. West African J Med. 2004;22:366-8.

5. Sutton EA. A Case of Myiasis of the Temporal Bone. J of the Royal Army Med Corps. 1926;47:613.

Cite this article as: Basavaraj $\mathrm{PK}$, Anandappa $\mathrm{MH}$, Sharma N, Karkala S, Prabhakaran V. Chronic otitis media progressing to temporal bone osteomyelitis and sequestration with mastoid cavity myiasis in paediatric age group: an unusual case report. Int $\mathbf{J}$ Otorhinolaryngol Head Neck Surg 2020;6:1001-4. 